pueden actuar como catalizador. En relación con el VIH/ SIDA, por ejemplo, podemos y debemos lograr la meta, " $3 \times 5$ ": tres millones de personas de los países en desarrollo sometidas a terapia antirretroviral para 2005.

Al mismo tiempo, la OMS debe forjar alianzas con otros líderes de la salud y otros interesados directos para reforzar la prevención de la infección por el VIH, de acuerdo con las metas mundiales acordadas. Me he comprometido en una guerra total contra la poliomielitis, y quiero llevar a término la erradicación de esta enfermedad durante mi mandato como Director General.

Aun con la mejor de las intenciones, los resultados son difíciles de prever. No obstante, los próximos años brindan una clara ventana de oportunidad para hacer grandes progresos en el campo de la salud. La importancia de la salud para el desarrollo económico y la seguridad mundial es hoy más obvia que nunca. Los líderes mundiales han tenido que dedicar una atención sin precedentes a cuestiones de salud, en particular, aunque no exclusivamente, a enfermedades infecciosas. Podemos y debemos utilizar esta oportunidad para impulsar un programa ambicioso en la salud pública internacional. Colaborando estrechamente con los países para medir los resultados sobre el terreno, garantizaremos que las aspiraciones mundiales se traduzcan en mejoras concretas para la salud y el bienestar de las comunidades.

21 de mayo de 2003

Entrevista realizada por Thomson Prentice, Director Gerente del Informe sobre la Salud en el Mundo.

\section{Prevención de la diabetes mellitus: un problema mundial}

Del 24 al 29 de agosto de 2003 se llevó a cabo el XVIII Congreso Anual de la Federación Internacional de Diabetes (IDF, por sus siglas en inglés) en París, Francia. A este evento asistieron alrededor de 15000 participantes de todo el mundo. Desde su origen, el crecimiento de este evento se relaciona con la importancia que esta enfermedad ha adquirido a escala mundial. De ser una enfermedad característica de los países desarrollados ha pasado a ser una epidemia en países en desarrollo y, a pesar de que existe un progreso considerable en el esclarecimiento de los mecanismos de sus causas y consecuencias, ${ }^{1}$ las estrategias para su prevención han resultado insuficientes. La Organización Mundial de la Salud estimó que para 2000 existirían en México 2.18 millones de personas con diabetes mellitus, ${ }^{2}$ sin embargo, la recientemente publicada Encuesta Nacional de Salud 2000, efectuada por el Instituto Nacional de Salud Pública, detectó 3.65 millones, ${ }^{3}$ y alrededor de 582826 mexicanos murieron de diabetes en el periodo 1980-2000. ${ }^{4}$ En México esta enfermedad se presentó, igual que en la mayoría de los países en desarrollo, de forma rápida y sin remplazar a los problemas pretransicionales como la desnutrición y las enfermedades infecto-contagiosas, lo que ha complicado todavía más la situación. ${ }^{5}$

No obstante que existen diversas intervenciones que han mostrado efectividad para prevenir la diabetes, la traducción de ese conocimiento en políticas y acciones efectivas ha sido posible sólo en contados casos. Uno de los países pioneros en la implantación de programas de salud efectivos para la prevención de enfermedades crónicas es Finlandia. Su Asociación Nacional de Diabetes ha desarrollado un plan 2000-2010 para la prevención y cuidado de la diabetes, que considera diversos sectores e identifica a los actores involucrados en cada acción clave para la prevención. ${ }^{6}$ De igual forma, el Programa de Prevención de Diabetes de los Estados Unidos de América ha mostrado resultados importantes que ahora deben traducirse en políticas públicas efectivas. ${ }^{7}$

En la reunión se hizo un recuento de múltiples acciones y estrategias aplicadas por diversos países para abordar este problema, entre las cuales destacan las siguientes: a) aumento del conocimiento sobre las oportunidades de prevención de la diabetes y sus complicaciones; $b$ ) promoción de un estilo de vida sano con énfasis en la actividad física y un plan alimentario saludable; c) detección efectiva y control de la diabetes mellitus; d) promoción del autocuidado en personas con diabetes; $\mathrm{e}$ ) atención especializada a niños con diabetes y apoyo necesario para sus familias; f) apoyo para centros de excelencia en investigación, educación y atención a la diabetes; g) promoción de independencia para personas con diabetes; $h$ ) reducción de las complicaciones de la diabetes; i) implantación de sistemas de información para monitorear la calidad de los servicios, y j) promoción de la colaboración internacional.

México, por la alta prevalencia de obesidad y diabetes, y por la creciente mortalidad causada por esta última, está obligado a un análisis profundo de las intervenciones efectivas que pueden ponerse en marcha lo antes posible, no únicamente en población de alto riesgo, sino a través de una estrategia dual que, además, fomente mayor conocimiento del problema en la población general.

Por último, vale la pena mencionar que el 14 de noviembre es el Día Mundial de la Diabetes, la principal campaña de concienciación sobre el problema en el mundo. Un 
tiempo de reflexión en el trabajo que se requiere para mejorar la prevención y el cuidado de las personas con esta enfermedad.

Dr. Simón Barquera. Departamento de Enfermedades Crónicas y Dieta,

Centro de Investigación en Nutrición y Salud, Instituto Nacional de Salud Pública.

\section{REFERENCIAS}

1. Haffner SM.Type 2 diabetes: Unravelling its causes and consequences: A compendium of classical papers. Cambridge: Medical
Publications, 2002.

2. N HM-DIA. Diabetes estimates 1995-2025 G inebra, Suiza:W orld Health 0 rganization, 2001.

3. 0 laiz G, Rojas R, Barquera S, Shamah T, A guilar $C, C$ ravioto $P$ et al. Encuesta $N$ acional de Salud 2000. Tomo 2. La salud de los adultos. Cuernavaca, Morelos, México: Instituto N acional de Salud Pública, 2003.

4. Barquera S,Tovar-G uzmán V, C ampos-N onato I, González-Villalpando C, Rivera-D ommarco J. Geography of diabetes mellitus mortality in Mexico:An epidemiologic transition analysis. Arch Med Res 2003. En prensa.

5. Rivera-D ommarco J, Barquera S, C ampirano F, Campos I, Safdie M,Tovar V.The epidemiological and nutritional transition in Mexico: Rapid

increase of non-communicable chronic diseases and obesity. Public Health N utr 2002; 5(1A):

113-122.

6. Finnish Diabetes Association. D evelopment programme for the prevention and care of diabetes in Finland 2000-2010. Finnish Diabetes Association-D iabetes C entre,Tampene, 2001. 7. Diabetes Prevention Program Research Group. Reduction in the incidence of type 2 diabetes with lifestyle inter vention or metformin. N Engl J Med 2002;346:393-403. 